\title{
Evolutionary many objective optimization based on bidirectional decomposition
}

\author{
LYU Chengzhong* and LI Weimin \\ Air and Missile Defense College, Air Force Engineering University, Xi' an 710051, China
}

\begin{abstract}
The decomposition based approach decomposes a multi-objective problem into a series of single objective subproblems, which are optimized along contours towards the ideal point. But non-dominated solutions cannot spread uniformly, since the Pareto front shows different features, such as concave and convex. To improve the distribution uniformity of non-dominated solutions, a bidirectional decomposition based approach that constructs two search directions is proposed to provide a uniform distribution no matter what features problems have. Since two populations along two search directions show differently on diversity and convergence, an adaptive neighborhood selection approach is presented to choose suitable parents for the offspring generation. In order to avoid the problem of the shrinking search region caused by the close distance of the ideal and nadir points, a reference point update approach is presented. The performance of the proposed algorithm is validated with four state-of-the-art algorithms. Experimental results demonstrate the superiority of the proposed algorithm on all considered test problems.
\end{abstract}

Keywords: many objective optimization, bidirectional decomposition, reference update, evolutionary algorithm.

DOI: $10.21629 / J S E E .2019 .02 .11$

\section{Introduction}

Simultaneous optimizations of multiple targets, which are defined as multi-objective problems (MOPs), are involved in many industrial and engineering applications [1-3]. Most problems among the MOPs conflict to others, and if one problem is optimized, another or more would become bad. Thus the solutions of the MOPs are a set of points which form an optimal front, and one is not superior to another on all problems of the MOPs when comparing any two solutions. For many years, researchers have been devoting themselves to finding an accurate and uniformly distributed approximation of the true optimal front.

Over past decades, a lot of multi-objective optimization algorithms (MOEAs) are suggested to solve the MOPs as

Manuscript received May 16, 2018.

*Corresponding author. their characteristics of fast convergence, well adaptability and simple mechanism. MOEAs are usually classified into three categories [4], which are Pareto based methods [5], indicator based methods [6] and decomposition based methods [7]. The representative method of decomposition based methods is MOEA based on decomposition (MOEA/D) [7]. It decomposes an MOP into a series of sub-problems, and each solution is optimized along a subproblem. This method could provide solutions spreading uniformly along the true Pareto front because of its decomposed thoughts.

The perfect performance of the MOEA/D attracts much attention of researchers who make a mass of contributions to studying the improved decomposition based methods. As the MOEA/D can measure the fitness improvement between an offspring solution and its parent, a number of multiple operator methods use it or its variants to evaluate the performance of operators and choose an appropriate operator for evolution. Multiple operator methods achieve a balance between the global search and the local search and bring superiority of each operator into full play during different search stages. The typical algorithms include the fitness-rate-rank-based multi-armed bandit (FRRMAB) [8], the adaptive composite operator selection and parameter control strategy for the MOEA/D (MOEA/D$\mathrm{CDE}$ ) [9], the adaptive differential evolution (DE) for MOPs (ADEMO/D) [10] and so on. A fundamental issue solved by multiple operator methods is called the exploration vs exploitation (EvE) dilemma [11], which is also an inevitable problem that all MOEAs have to face in the process of their improvement.

It is a good choice to use a multiple operator method for the EvE dilemma in MOPs, but it is not effective in many objective problems. With the increasing number of objectives, the solutions get sparse and almost all solutions in a population are non-dominated. In addition, a high dimensional objective decision causes the search scope to increase dramatically. Thus, the EvE dilemma becomes the 
focus of the attention in many objective problems again. Deb et al. [12] proposed a reference-point based many objective NSGA-II (NSGA-III) in which the maintenance of the diversity among population members is aided by supplying and adaptively updating a number of well-spread reference points, and this update method is somewhat similar to the MOEA/D. To enhance the convergence, Yuan et al. [13] suggested a new dominance relation in the basis of NSGA-III, which was named as $\theta$-DEA. In this algorithm, the non-dominated sorting scheme based on the new dominance relation is employed to rank solutions in the environmental selection phase, ensuing both convergence and diversity. To balance the convergence and diversity, Li et al. [14] presented an evolutionary MOEA based on the dominance and decomposition (MOEA/DD), in which a new systematic approach of integrating dominance and decomposition alloted widely spread reference objective vectors with a unique sub-region in the objective space. Wu et al. [15] developed an adversarial decomposition method that leveraged the complementary characteristics of two different scalarizing functions within a single paradigm.

Most of the MOEA/D variants are used to solve many objective problems and improve the generation of reference points or vectors which play a crucial role in the environmental selection. To improve the convergence and diversity of the decomposition based algorithms in many problems, a new decomposed based MOEA is presented, which is named evolutionary MOEA based on bidirectional decomposition (MOEA/BD). In this method, a bidirectional selection pressure to two co-evolving populations is provided to enhance the convergence and diversity simultaneously. As the distribution of the two co-evolving populations shows during the search process, an adaptive neighborhood selection approach is provided to choose parents. In order to avoid the problem of the shrinking search region caused by the close distance of the ideal and nadir points, we present a reference point update approach. To validate the effectiveness of the MOEA/BD, five wellknown DTLZ test problems [16] are adopted. The comparative experiments are demonstrated when comparing the proposed algorithm with four state-of-the-art MOEAs, e.g. many-objective optimization algorithm based on adversarial decomposition (MOEA/D-AD) [15], NSGA-III [12], improved metaheuristic based on the $\mathrm{R} 2$ indicator for many objective optimization (MOMBI-II) [17] and MOEA/D [7].

The rest of this paper is organized as follows. Some preliminaries are provided in Section 2. The detailed description of the MOEA/BD is given in Section 3. Section 4 analyzes the results of comparison experiments. Finally, conclusions are summarized and further work along the direction of the adaptive selection is discussed in Section 5.

\section{Preliminaries}

\subsection{Basic definitions}

The mathematic description of multi-objective optimization problems for the minimization is described as follows [18]:

Minimize:

$$
F(\boldsymbol{x})=\left\{f_{1}(x), f_{2}(x), \ldots, f_{m}(x)\right\}
$$

where $\boldsymbol{x}=\left(x_{1}, x_{2}, \ldots, x_{n}\right) \in \boldsymbol{\Omega} \subseteq \mathbf{R}^{n}$ is an $n$ dimensional decision vector; $\boldsymbol{\Omega}$ is the decision space; $m$ is the objective number; $F: \boldsymbol{\Omega} \rightarrow \boldsymbol{\Theta} \subseteq \mathbf{R}^{m}$ consists of a set of $m$ objective functions, and is a mapping from $\Omega$ to the objective space $\Theta[18]$.

Definition 1 Let $\boldsymbol{x}, \boldsymbol{y} \in \boldsymbol{\Omega}, \boldsymbol{x}$ is said to the Pareto dominate $\boldsymbol{y}$, which is donated by $\boldsymbol{x} \prec \boldsymbol{y}$, iff $f_{i}(\boldsymbol{x}) \leqslant f_{i}(\boldsymbol{y})$ for each $i \in\{1, \ldots, m\}$ and $f_{j}(\boldsymbol{x})<f_{j}(\boldsymbol{y})$ for at least one index $j \in\{1, \ldots, m\}$.

Definition 2 A point $\boldsymbol{x} \in \boldsymbol{\Omega}$ is the Pareto optimal iff there is no $\boldsymbol{x}^{*} \in \boldsymbol{\Omega}$ such that $\boldsymbol{x}^{*} \prec \boldsymbol{x}$.

Definition 3 The set of all Pareto optimal points is the Pareto set (PS), which is donated by

$$
P S:=\{\boldsymbol{x} \in \boldsymbol{\Omega} \mid \neg \exists \boldsymbol{y} \in \boldsymbol{\Omega}, \boldsymbol{y} \prec \boldsymbol{x}\} .
$$

Definition 4 The set of all Pareto optimal objective vectors is called Pareto front (PF), donated by

$$
P F:=\{\boldsymbol{y}=F(\boldsymbol{x}) \mid \boldsymbol{x} \in \boldsymbol{P} \boldsymbol{S}\} .
$$

\subsection{Modified Tchebycheff decomposition}

Under some mild conditions, the task of approximating the PF can be decomposed into several scalar optimization sub-problems, each of which is formed by a weighted aggregation of all individual objectives [8]. There are some established approaches for constructing scalarizing functions. We choose one of Tchebycheff decomposition approaches, the modified Tchebycheff decomposition (mTCH) [19], which is validated to be more efficient than the generalized decomposition. More formally, the optimization problem of the m-TCH is in the form:

Minimize:

$$
\begin{aligned}
g\left(\boldsymbol{x} \mid \boldsymbol{w}, \boldsymbol{z}^{*}\right)= & \max _{1 \leqslant i \leqslant m}\left\{\left|f_{i}(\boldsymbol{x})-z_{i}^{*}\right| / w_{i}\right\} \\
& \text { s.t. } \boldsymbol{x} \in \boldsymbol{\Omega}
\end{aligned}
$$

where $i \in\{1,2, \ldots, m\}, z^{*}=\left(z_{1}^{*}, z_{2}^{*}, \ldots, z_{m}^{*}\right)$ is the idea point and $z_{i}^{*}=\min \left(f_{i}(\boldsymbol{x}) \mid \boldsymbol{x} \in \boldsymbol{\Omega}\right), \boldsymbol{w}=\left(w_{1}, \ldots, w_{m}\right) \in$ $\boldsymbol{\Lambda}$ is a unique weight vector, where $\boldsymbol{\Lambda}$ is the set of all weight vectors.

\section{The proposed algorithm}

In this section, we introduce the details of the MOEA/BD. After initialization, the population is copied and the popu- 
lation size is $2 N$. Two completely same populations are defined as forward directed population $P_{1}$ (size $N$ ) and backward directed population $P_{2}($ size $N) . P_{1}$ and $P_{2}$ are coevolving populations and share the same weight vectors. The same weight vectors used in different scalarizing functions provide different search directions. As the scalarizing functions are a little different, one performs better on convergence, and the other has a better diversity. Then the two populations evolve with each other and retain solutions according to their scalarizing functions. Finally, reference points are updated to locate an accurate region for the next search stage. The major components of the MOEA/BD are explained in the following subsections.

\subsection{Bidirectional decomposition}

The bidirectional decomposition possesses two search directions. One is the direction that all solutions in $P_{1}$ aggregate to the ideal point, and the other is that all solutions in $P_{2}$ are pushed far away from the nadir point. As the references are different, we improve the TCH to fit the search directions of $P_{1}$ and $P_{2}$.

As different problems have various scales of objectives, the objective values need to be normalized before using the m-TCH.

$$
f_{i}^{\prime}(\boldsymbol{x})=\frac{f_{i}(\boldsymbol{x})-z_{i}^{*}}{z_{i}^{\text {nad }}-z_{i}^{*}}
$$

where $z_{i}^{\text {nad }}=\max _{\boldsymbol{x} \in \boldsymbol{\Omega}}\left\{\left(f_{i}(\boldsymbol{x})\right)\right\}, i \in\{1, \ldots, m\}$.

The adapted $\mathrm{m}-\mathrm{TCH}$ approach used in $P_{1}$ is defined as follows:

$$
\begin{gathered}
g^{1}\left(\boldsymbol{x} \mid \boldsymbol{w}, \boldsymbol{z}^{*}\right)=\max _{1 \leqslant i \leqslant m}\left\{\left(f_{i}^{\prime}(\boldsymbol{x})-z_{i}^{*}\right) / w\right\}+ \\
\alpha \sum_{i=1}^{m}\left(f_{i}^{\prime}(\boldsymbol{x})-z_{i}^{*}\right) / \boldsymbol{w}
\end{gathered}
$$

where $\alpha$ is an augmentation coefficient, and suggested to be a sufficiently small value $\alpha=10^{-6}$ [19].

The scalarizing function used in $P_{2}$ is defined as follows:

$$
\begin{gathered}
g^{2}\left(\boldsymbol{x} \mid \boldsymbol{w}, \boldsymbol{z}^{\mathrm{nad}}\right)=\max _{1 \leqslant i \leqslant m}\left\{\left(f_{i}^{\prime}(\boldsymbol{x})-z_{i}^{\mathrm{nad}}\right) / \boldsymbol{w}\right\}+ \\
\alpha \sum_{i=1}^{m}\left(f_{i}^{\prime}(\boldsymbol{x})-z_{i}^{\mathrm{nad}}\right) / \boldsymbol{w} .
\end{gathered}
$$

Compared with the m-TCH in (4), the augmentation term is added to help avoid weakly Pareto optimal solutions. Additionally, the absolute operator is removed to allow $f_{i}^{\prime}(\boldsymbol{x})$ exceed the region $\left[z_{i}^{*}, z_{i}^{\text {nad }}\right]$. Since the direction of $P_{2}$ is against to that of $P_{1}$, the ideal point is replaced by the nadir point.
The bidirectional decomposition illustration is shown in Fig. 1. Suppose that the original point is the ideal point and the nadir point is located at the top right of Fig. 1. The solid line denotes that all points in $P_{1}$ pushed toward the ideal point and the intersections of the solid line and curve are solutions of $P_{1}$. Similarly, the dotted line is the search direction of $P_{2}$ that all points are pushed away from the nadir point, and the intersections of the dotted line and curve are solutions of $P_{2}$. Two populations share the same weight vectors, but the search directions generated by different scalarizing functions with these weight vectors are different. It is obvious that the diversity of solutions found by one scalarizing function is not as good as that of the bidirectional decomposition. Two search directions achieve complementary effects.

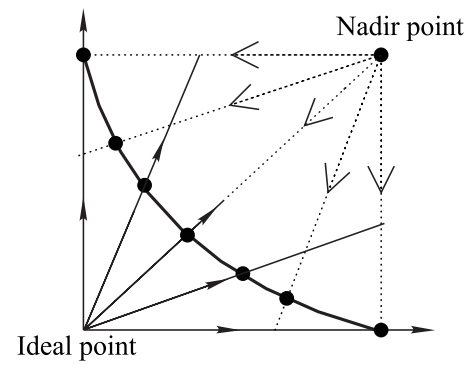

Fig. 1 Illustration of bidirectional decomposition

\subsection{Mating neighbor selection}

In the MOEA/D, neighbors of the weight vector $\boldsymbol{w}_{i}$ are defined as a set of its several closest weight vectors denoted as $\left\{w_{i_{1}}, \ldots, w_{i_{T}}\right\}$, where $T$ is the number of the closest weight vectors and $i \in\{1, \ldots, N\}$. Thus, neighbors of solution $\boldsymbol{x}_{i}$ are defined as a set of solutions to subproblems with $\left\{w_{i_{1}}, \ldots, w_{i_{T}}\right\}$. But two co-evolving populations share the same weight vectors in the MOEA/BD. Each solution has two sets of neighbors spreading differently. Thus, an adaptive selection method, named probability matching (PM) [20], is used to choose suitable mating neighbors.

Assume that the solution $\boldsymbol{x}_{i}$ is optimized at the time point $t$ and the mating neighbor pool is $\boldsymbol{S}=$ $\left\{s^{1}, s^{2}\right\}$, where $\boldsymbol{s}^{1}=\left\{x_{i_{1}}^{1}, \ldots, x_{i_{T}}^{1}\right\} \subseteq \boldsymbol{P}_{1}$ and $\boldsymbol{s}^{2}=$ $\left\{x_{i_{1}}^{2}, \ldots, x_{i_{T}}^{2}\right\} \subseteq \boldsymbol{P}_{2}$. Let $\boldsymbol{Q}_{j, t}$ and $\boldsymbol{q}_{j, t+1}$ be the probability vector and estimate of $s^{j}$ reward respectively.

$$
\begin{gathered}
\widehat{\boldsymbol{q}}_{j, t+1}=(1-\beta) \times \widehat{q}_{j . t}+\beta \times r \\
\boldsymbol{Q}_{j, t+1}=p_{\min }+\left(1-2 \times p_{\min }\right) \times \frac{\widehat{q}_{j, t+1}}{\sum_{k=1}^{2} \widehat{q}_{k, t+1}}
\end{gathered}
$$

where $r$ is the reward of the selected mating neighbor set, $\boldsymbol{x}_{\text {old }}$ is the parent individual of the sub-problem $i-1, \boldsymbol{x}_{\text {new }}$ is the individual that replaces $\boldsymbol{x}_{\text {old }}$ and $\beta$ is the adaptation 
rate. In this method, the worst probability is $p_{\min }$ and the best probability is $Q_{\max }=1-p_{\min }$. As recommended by the developer, $p_{\min }$ and $\beta$ are set to 0.2 and 0.8 , respectively.

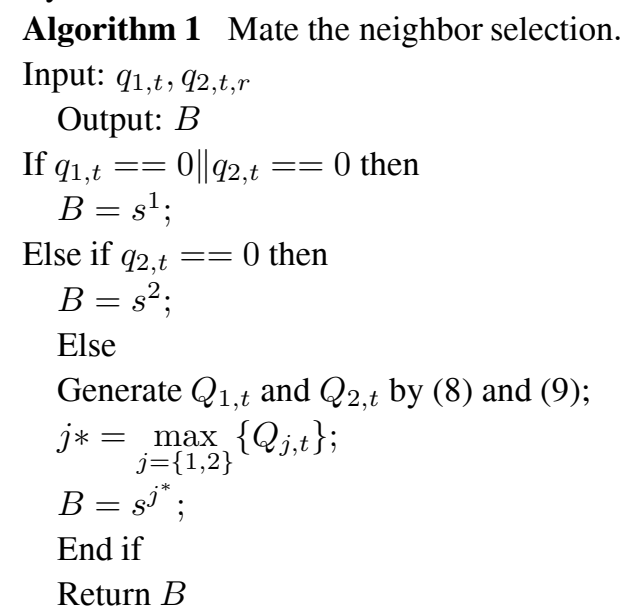

\subsection{Reference point update}

A problem with the normalization is that the feasible region scales go down when the ideal and nadir points are close. Then, a poor distribution of solutions emerges and the proposed algorithm loses diversity. To overcome the drawback, we update the nadir point with a smart way.

We discuss three conditions under which the nadir point needs to be updated with a new way. Condition 1 is that the ideal and nadir points are close. Condition 2 is that a new boundary point is generated. Condition 3 is that the nadir point is not updated during $D$ generations. If none of the conditions are met, the nadir point is updated as mentioned in [7]. A slight change of reference points has a great influence on accuracy and distribution; the tolerance threshold $\varepsilon$ is set to 0.01 when all conditions are normalized. Another parameter is the length of the reference point record $D$, which is set to 5 as suggested in [17]. The pseudo code of the updated reference point is shown in Algorithm 2.

Algorithm 2 Update the reference point.

$$
\text { Input: } \boldsymbol{z}^{*}, \boldsymbol{z}^{\text {nad }}, \boldsymbol{P}^{*}, \text { the size of } \boldsymbol{P}^{*} N
$$

Output: $z^{*}, z^{\text {nad }}$

$$
\text { For } d=1 \text { to } D-1 \text { do }
$$$$
L(d)=L(d+1) \text {; }
$$

End for

$$
L(D)=z^{\text {nad }}
$$

Obtain the vector of variances $\boldsymbol{v}$ for $\boldsymbol{z}^{\text {nad }}$ from $L$;

$$
\begin{aligned}
& \text { For } i=1 \text { to } m \text { do } \\
& z_{i}^{*}=\min \left\{z_{i}^{*}, z_{i}^{\text {min }}\right\} ; \\
& \text { If }\left|z_{i}^{*}-z_{i}^{\text {nad }}\right| / z_{i}^{\text {nad }}<\varepsilon \text { then } \\
& \quad z_{i}^{\text {nad }}=\max _{i \in\{1, \ldots, m\}}\left\{z_{i}^{\text {nad }}\right\} ;
\end{aligned}
$$

Else if $\left(z_{i}^{\max }-z_{i}^{\text {nad }}\right) / z_{i}^{\text {nad }}>\varepsilon$ then

$$
z_{i}^{\text {nad }}=2 z_{i}^{\max }-z_{i}^{\text {nad }}
$$

Else if $v_{i}==0$ then

Get the maximum value $z_{i}^{\#}$ for $z_{i}^{\text {nad }}$ from $L$;

$$
z_{i}^{\text {nad }}=\left(z_{i}^{\text {nad }}+z_{i}^{\#}\right) / 2 \text {; }
$$

Else

$$
z_{i}^{\text {nad }}=\max \left\{z_{i}^{\text {nad }}, z_{i}^{\max }\right\}
$$

End if

End for

Return $\boldsymbol{z}^{*}, \boldsymbol{z}^{\text {nad }}$

\subsection{Population update}

After the mating neighbors are ensured, another parent individual is randomly selected from the mating pool. The new offspring is compared with the parent according to the $\mathrm{BD}$ and replaces $T$ individuals if it is better than them. The pseudo code of the MOEA/BD is given in Algorithm 3.

The non-dominated sorting of a population of size $N$ requires $O\left(N \log _{10} N\right)$ computations. The updated reference needs $O(N)$ computations. After the population is duplicated in two, the evolution begins. The generation of new individuals of a population of size $2 N$ requires $O(N)$ computations. All operations of population update including sub-problem value calculation and individual reservation require $O(N)$ computations. The overall complexity of the proposed algorithm is $O\left(N \log _{10} N\right)$.

Algorithm 3 Procedure of MOEA/BD.

Input: algorithm parameters

Output: $\boldsymbol{P}^{*}$

$\boldsymbol{\Lambda} \leftarrow$ Generate reference vectors();

$\boldsymbol{P}_{0} \leftarrow$ Initialize population();

$\left(\boldsymbol{z}^{*}, \boldsymbol{z}^{\text {nad }}\right) \leftarrow$ Initialize reference points () ;

$t \leftarrow 0$;

$\boldsymbol{P}_{1} \leftarrow \boldsymbol{P}_{0}, \boldsymbol{P}_{2} \leftarrow \boldsymbol{P}_{0} ;$

While the termination criterion is not met do

For $i=1$ to $2 N$ do

$B \leftarrow$ Mating neighbors selection $\left(q_{1, t}, q_{2, t}, r\right)$;

Randomly select a parent $\boldsymbol{x}^{\prime}$ from $B$;

Generate new solution $\boldsymbol{y}$ with $x_{i}$ and $\boldsymbol{x}^{\prime}$; $\left(\boldsymbol{z}^{*}, \boldsymbol{z}^{\text {nad }}\right) \leftarrow$ Reference point update $\left(\boldsymbol{z}^{*}, \boldsymbol{z}^{\text {nad }}\right.$, $\left.\boldsymbol{P}^{*}, N\right)$

For $j=1$ to 2 do

While $c<T$

Randomly pick a solution from $s^{j}$;

$r=0$; $\eta=\left(g^{j}(a)-g^{j}(y)\right) / g^{j}(a)$

If $g^{j}(y)<g^{j}(a)$ do

Replace $\boldsymbol{y}$ with $\boldsymbol{a}$;

Remove $x_{i}$ from $s^{j}$; $r=\max \{r, \eta\}$;

\section{End if}

c++

End while 


\author{
End for \\ End for \\ End while \\ Return $\boldsymbol{P}^{*}$
}

\section{Experimental studies}

\subsection{Benchmark functions}

For the purpose of performance comparisons, five test problems from the DTLZ test suite [16] (DTLZ1 to DTLZ5) are chosen for our empirical studies. The objective number $m$ is set to 3,5 and 10 . The number of decision variables $n$ is set to $n=m+k+1$ for DTLZ test problems, where $k=5$ for DTLZ1 and $k=10$ for DTLZ2 to DTLZ5. The DTLZ test suite has various characteristics including linear, convex, concave mixed multimodal and so on, and is widely used to validate the performance of MOEAs.

\subsection{Performance measure}

Two performance measures are used to assess the performance of the proposed algorithm and its comparisons, which are the inverted generational distance (IGD) [21].

IGD: Let $\boldsymbol{P}^{*}$ be a set of solutions distributed in the true Pareto-optimal front, which is a sphere composed of all best solutions. Let $\boldsymbol{P}$ be an approximation set got by the MOEA. The IGD metrics of $\boldsymbol{P}$ are obtained by

$$
\operatorname{IGD}\left(\boldsymbol{P}, \boldsymbol{P}^{*}\right)=\frac{\sum_{\boldsymbol{x} \in \boldsymbol{P}^{*}} \operatorname{dist}(\boldsymbol{x}, \boldsymbol{P})}{\left|\boldsymbol{P}^{*}\right|}
$$

where $\operatorname{dist}(\boldsymbol{x}, \boldsymbol{P})$ is the minimum Euclidean distance between $\boldsymbol{x}$ and its nearest solution in $\boldsymbol{P}$, and $\left|\boldsymbol{P}^{*}\right|$ is the number of elements in $\boldsymbol{P}^{*}$.

\subsection{Experimental settings}

Four state-of-the-art MOEAs, including MOEA/D, NSGA-III, MOMBI-II and MOEA/AD are considered for comparisons. The parameter settings for experiments are listed as follows.

(i) Population size: according to [12], the population size in NSGA-III is set as the smallest multiple of the four larger than the number of reference points $(H)$ produced by the two layer weight vector generation method. Since MOMBI-II involves a binary tournament selection, it requires the population size to be even. Therefore, the population size of the MOMBI-II is set as NSGA-III. The population size of MOEA/D and MOEA/AD is set to $N=H$. The population sizes of all algorithms for different objective numbers are shown in Table 1.

(ii) Number of independent runs and termination: each algorithm runs 20 times independently on each test problem. The stop termination condition is a predefined number of generations. The max generation is set to 200, 400 and 600 when the objective number is 3,5 and 10 , respectively.

Table 1 Number of weight vectors and population size

\begin{tabular}{cccc}
\hline$m$ & $H$ & $\begin{array}{c}\text { NSGA-III } \\
\text { MOMBI-II }\end{array}$ & $\begin{array}{c}\text { MOEA/D } \\
\text { MOEA/AD }\end{array}$ \\
\hline 3 & 91 & 92 & 91 \\
5 & 210 & 212 & 210 \\
10 & 275 & 276 & 275 \\
\hline
\end{tabular}

(iii) Parameter setting for operators: the operator used to generate the offspring is the simulated binary crossover (SBX) and the polynomial-based mutation [22]. The crossover probability $p c$ and mutation probability $\mathrm{pm}$ are set to 1.0 and 20 . The mutation rate is set to $1 / n$ and its distributed index is set to 20 .

(iv) Parameter setting for algorithm: all parameters used in the MOEA/D, MOEA/AD, NSGA-III, MOMBIII are recommended by their developers. A penalty parameter is set to 5.0 in the MOEA/AD. In both MOEA/AD and MOEA/D, the neighborhood size $T$ is set to 20. In the MOMBI-II, two parameters are set as $\varepsilon=1 \mathrm{e}-3$ and $\alpha=0.5$.

\subsection{IGD indicator analysis}

The comparison results of the MOEA/BD with other four MOEAs in terms of mean IGD values are presented in Table 2. The best metric values are highlighted in bold face with the gray background.

In DTLZ1 to DTLZ4 test problems with 3- and 5objective, the results of five MOEAs are similar. The MOEA/BD gets a little better result than the other MOEAs in most test problems, except in DTLZ4 and DTLZ1 with 3-objective. The results among DTLZ1 to DTLZ5 with 10-objective are obvious different. Except 10-objective DTLZ3, the MOEA/BD gets better results than the other MOEAs in all 10-objective cases. DTLZ5 is a more complicated problem as the PF is unclear with the objective number increasing [23]. NSGA-III obtains better performance than the other MOEAs in the DTLZ5 test problem with 3-objective, and MOMBI-II performs only worse than NSGA-III in this case. The MOEA/BD gets the best IGD values in 5- and 10-objective cases. Based on the above experimental results regarding the IGD, it is clear that our proposed algorithm shows the best performance in 11 out of 15 cases.

Two main statements can be derived from the present comparison. First, no matter the PFs of problems show concave or convex, the BD method provides uniformly distributed solutions on the hyper-plane as two opposite search directions are given. Second, the reference extends the search space by updating the nadir point location when the search space is small or the optimization gets into the local optimum. 
Table 2 Comparison results on DTLZ regarding IGD

\begin{tabular}{|c|c|c|c|c|c|c|}
\hline Problem & $m$ & MOMB-III & MOEAD & NSGA-III & MOEA/AD & MOEA/BD \\
\hline \multirow{3}{*}{ DTLZ1 } & 3 & $1.0739 \mathrm{E}-02$ & $1.0726 \mathrm{E}-02$ & $1.0725 \mathrm{E}-02$ & $1.0725 \mathrm{E}-02$ & $1.0725 \mathrm{E}-02$ \\
\hline & 5 & $5.2765 \mathrm{E}-02$ & $5.2714 \mathrm{E}-02$ & $5.2715 \mathrm{E}-02$ & $5.2710 \mathrm{E}-02$ & $5.2708 \mathrm{E}-02$ \\
\hline & 10 & $1.9255 \mathrm{E}-01$ & $9.6003 \mathrm{E}-02$ & $9.8537 \mathrm{E}-02$ & $9.8363 \mathrm{E}-02$ & $9.7704 \mathrm{E}-02$ \\
\hline \multirow{3}{*}{ DTLZ2 } & 3 & $2.8559 \mathrm{E}-02$ & $2.8549 \mathrm{E}-02$ & $2.8549 \mathrm{E}-02$ & $2.8549 \mathrm{E}-02$ & $2.8548 \mathrm{E}-02$ \\
\hline & 5 & $1.6518 \mathrm{E}-01$ & $1.6514 \mathrm{E}-01$ & $1.6513 \mathrm{E}-01$ & $1.6514 \mathrm{E}-01$ & 1.6512E-01 \\
\hline & 10 & $3.7531 \mathrm{E}-01$ & $3.7430 \mathrm{E}-01$ & $3.7564 \mathrm{E}-01$ & $3.7430 \mathrm{E}-01$ & 3.7331E-01 \\
\hline \multirow{3}{*}{ DTLZ3 } & 3 & $2.8591 \mathrm{E}-02$ & $2.8553 \mathrm{E}-02$ & $2.8551 \mathrm{E}-02$ & $2.8554 \mathrm{E}-02$ & $2.8550 \mathrm{E}-02$ \\
\hline & 5 & $1.6539 \mathrm{E}-01$ & $1.6526 \mathrm{E}-01$ & $1.6522 \mathrm{E}-01$ & $1.6519 \mathrm{E}-01$ & $1.6514 \mathrm{E}-01$ \\
\hline & 10 & $4.5276 \mathrm{E}-01$ & $3.7603 \mathrm{E}-01$ & 4.2979E-01 & 3.7427E-01 & 4.0133E-01 \\
\hline \multirow{3}{*}{ DTLZ4 } & 3 & $2.8566 \mathrm{E}-02$ & $2.8548 \mathrm{E}-02$ & $2.8556 \mathrm{E}-02$ & 2.8549E-02 & $2.8555 \mathrm{E}-02$ \\
\hline & 5 & $1.6546 \mathrm{E}-01$ & $1.9545 \mathrm{E}-01$ & $1.6515 \mathrm{E}-01$ & $1.6514 \mathrm{E}-01$ & 1.6512E-01 \\
\hline & 10 & $3.9622 \mathrm{E}-01$ & $5.4337 \mathrm{E}-01$ & $3.7335 \mathrm{E}-01$ & $3.7431 \mathrm{E}-01$ & $3.7276 \mathrm{E}-01$ \\
\hline \multirow{3}{*}{ DTLZ5 } & 3 & $7.8736 \mathrm{E}-03$ & $1.7711 \mathrm{E}-02$ & $4.0544 \mathrm{E}-03$ & $1.6214 \mathrm{E}-02$ & $1.4944 \mathrm{E}-02$ \\
\hline & 5 & $2.4021 \mathrm{E}-01$ & $3.4838 \mathrm{E}-01$ & $1.2293 \mathrm{E}-01$ & $1.1300 \mathrm{E}-01$ & $2.2730 \mathrm{E}-02$ \\
\hline & 10 & $6.6814 \mathrm{E}-01$ & $1.5604 \mathrm{E}-01$ & $4.8070 \mathrm{E}-01$ & $2.0572 \mathrm{E}-01$ & $1.8394 \mathrm{E}-02$ \\
\hline
\end{tabular}

\subsection{Box plot analysis}

Since some IGD results in Table 1 are so similar that comparisons are not clear, we use the well-known box plot as a statistical method for graphically depicting differences among groups of data. All IGD values obtained in the 20 runs are plotted on the boxplot.

Fig. 2 shows box plots for DTLZ1 to DTLZ5 with different objectives. The red lines in each box plot are median values of results achieved by an MOEA in 20 runs. The symbol "+" denotes an outlier. The comparisons in DTLZ1 and DTLZ2 revealed by the box plot are the same as that in the IGD indicator. However, the median value shows that all MOEAs perform similarly, but the result obtained by the MOEA/BD shown in the box plot is different from the mean value result in Table 2 . There is an outlier that seriously affects the statistical result. Similar phenomena also occur in DTLZ4 with 5-objective, where the MOEA/D gets the similar result to the other four if there are no outliers. The box plot reveals a visualized comparison results. NSGA-III obtains a better result than the other algorithms, and the MOEA/BD performs much better than the other four MOEAs in the DTLZ5 test problems with 5- and 10objective.

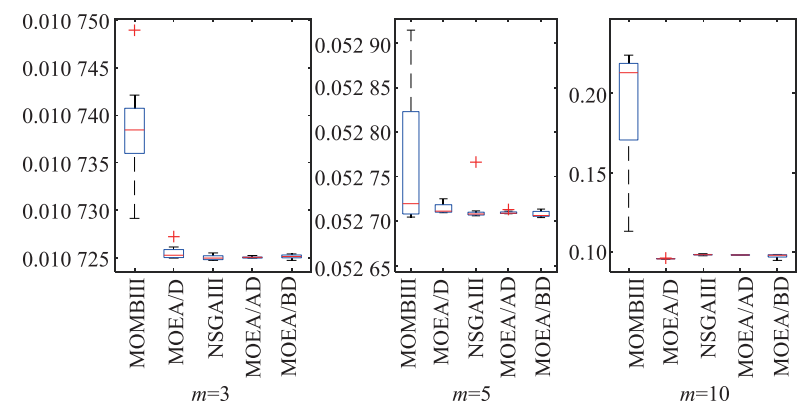

(a) DTLZ1

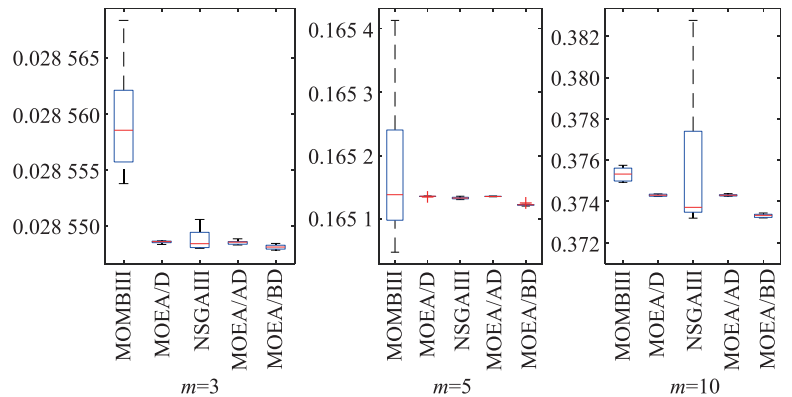

(b) DTLZ2

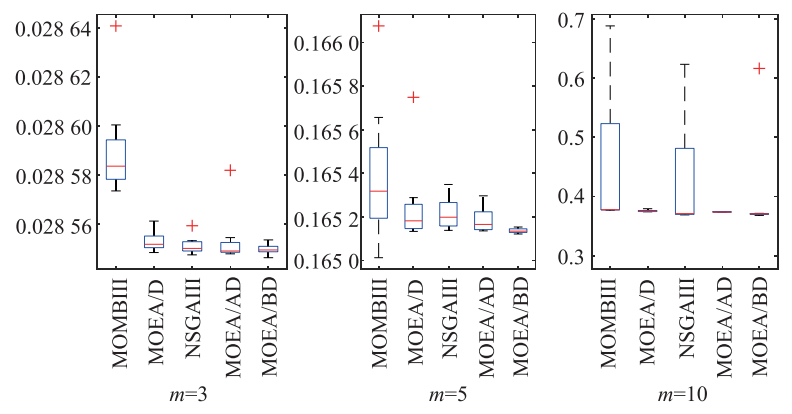

(c) DTLZ3

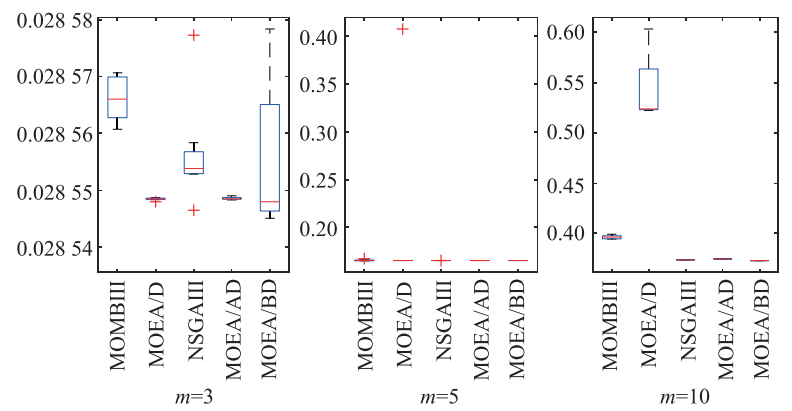

(d) DTLZ4 


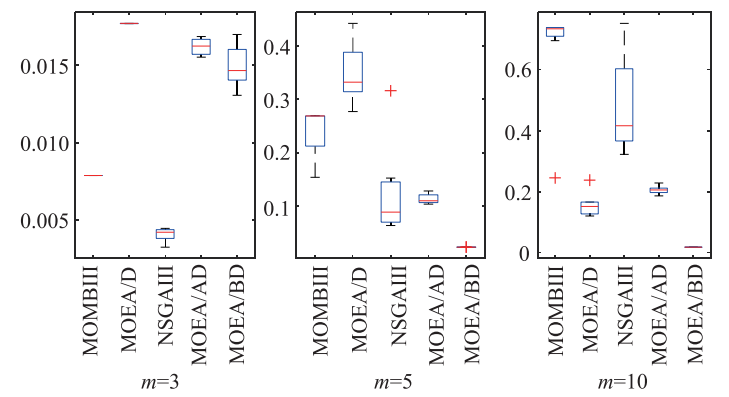

(e) DTLZ5

Fig. 2 Box plots for DTLZ1 to DTLZ5 with different objectives

\subsection{Further discussions}

In order to further investigate the performance of the MOEA/BD in more objective problems, diversity comparisons in test problems with 20-objective are given. The number of weight vectors $\boldsymbol{H}$ is set to 230 and the population size $N$ is set to 230 for the MOEA/D and MOEA/AD, and 232 for the NSGA-III and MOMOBI-II. Fig. 3 shows the parallel coordinates of non-dominated fronts got by five MOEAs in the DTLZ1 test problem. These plots clearly show that the MOEA/BD obtains a well converged and widely distributed set of non-dominated solutions in DTLZ1 test problem with 20-objective. Although the nondominated solutions achieved by NSGA-III is also well converged, the distribution uniformity is not as good as the MOEA/BD. In contrast, the non-dominated fronts obtained by the MOEA/D and the MOEA/AD do not converge well but are better than that got by MOMBI-II.

It is clear that all decomposition based methods in the experiments are better than MOMBI-II, an indicator based method. Although the MOEA/D and MOEA/AD perform not as good as NSGA-III and MOEA/BD, there are no missing objective dimensions. If the generations are enough, the performance between the four MOEAs will not be obvious. Thus, the MOEA/D and MOEA/AD are worse than the MOEA/BD on convergence, especially when the objective dimension is increasing. MOEA/BD is better than NSGA-III on distribution. This is probably caused by the bidirectional decomposition which provides a more accurate region to search.

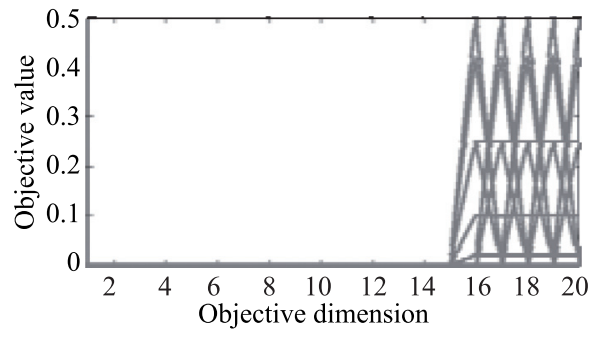

(a) MOMBI-II

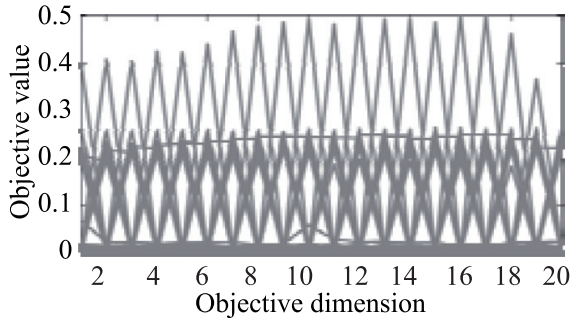

(b) MOEAD

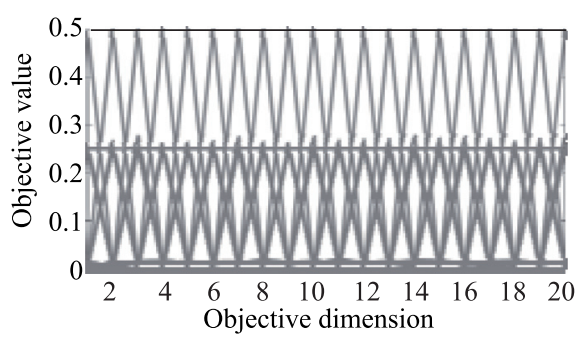

(c) NSGA-III

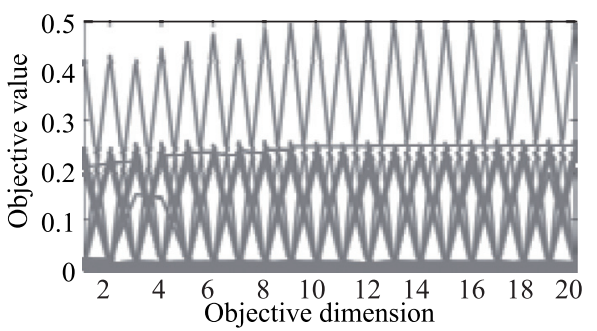

(d) MOEA/AD

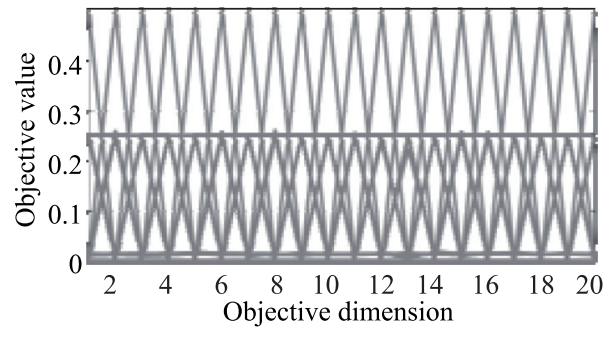

(e) MOEA/BD

Fig. 3 Parallel coordinates of non-dominated fronts got by five MOEAs in DTLZ1 test problem with 20-objective

\section{Conclusions}

This paper proposes the bidirectional decomposition based approach that constructs two search directions to provide a uniform distribution no matter what features problems have. Since two populations along two search directions show different diversity and convergence, an adaptive neighborhood selection approach is provided to choose parents. In order to avoid the problem of shrinking the search region caused by the close distance of the ideal and nadir points, we present a reference point update approach. The performance of our proposed algorithm is validated with four state-of-the-art algorithms. Experimental results demonstrate the superiority of the proposed algorithm on all considered test problems. In the future work, three or 
more search directions based on the decomposition are the potential and valuable problem to be investigated.

\section{References}

[1] LI J T, ZHANG S, LIU X L, et al. Multi-objective evolutionary optimization for geostationary orbit satellite mission planning. Journal of Systems Engineering and Electronics, 2017, 28(5): $934-945$.

[2] LIU A J, PFUND M, FOWLER J. Scheduling optimization of task allocation in integrated manufacturing system based on task decomposition. Journal of Systems Engineering and Electronics, 2016, 27(2): $422-433$.

[3] LI K L, WANG J. Multi-objective optimization for cloud task scheduling based on the ANP model. Chinese Journal of Electronics, 2017, 26(5): 889-898.

[4] WANG Z K, ZHANG Q F, ZHOU A M, et al. Adaptive replacement strategies for MOEA/D. IEEE Trans. on Cybernetics, 2016, 46(2): 474-486.

[5] DEB K, AGRAWAL S, PRATAP A, et al. A fast elitist nondominated sorting genetic algorithm for multi-objective optimisation: NSGA-II. Proc. of the 6th International Conference on Parallel Problem Solving from Nature, 2000: 849-858.

[6] VILLALOBOS C A R, COELLO C A. A new multiobjective evolutionary algorithm based on a performance assessment indicator. Proc. of the 14th International Conference on Genetic and Evolutionary Computation, 2012: 505-512.

[7] ZHANG Q F, LI H. MOEA/D: a multiobjective evolutionary algorithm based on decomposition. IEEE Trans. on Evolutionary Computation, 2007, 11(6): 712-731.

[8] LI K, FIALHO A, KWONG S, et al. Adaptive operator selection with bandits for a multiobjective evolutionary algorithm based on decomposition. IEEE Trans. on Evolutionary Computation, 2014, 18(2): 114-130.

[9] LIN Q Z, LIU Z W, YAN Q, et al. Adaptive composite operator selection and parameter control for multiobjective evolutionary algorithm. Information Sciences, 2016, 339: 332 - 352.

[10] VENSKE S M, GONÇALVES R A, DELGADO M R. ADEMO/D: multiobjective optimization by an adaptive differential evolution algorithm. Neurocomputin, 2014, 127(2): $65-77$.

[11] KAELBLING L P. Associative reinforcement learning: a generate and test algorithm. Machine Learning, 1994, 15(3): $299-$ 319.

[12] DEB K, JAIN H. An evolutionary many-objective optimization algorithm using reference-point-based nondominated sorting approach, part I: solving problems with box constraints. IEEE Trans. on Evolutionary Computation, 2014, 18(4): 577-601.

[13] YUAN Y, XU H, WANG B, et al. A new dominance relation based evolutionary algorithm for many-objective optimization. IEEE Trans. on Evolutionary Computation, 2016, 20(1): 1637.

[14] LI K, DEB K, ZHANG Q, et al. An evolutionary manyobjec- tive optimization algorithm based on dominance and decomposition. IEEE Trans. on Evolutionary Computation, 2015, 19(5): $694-716$

[15] WU M Y, LI K, KWONG W, et al. Evolutionary manyobjective optimization based on adversarial decomposition. IEEE Trans. on Cybernetics, 2018: 1-24

[16] DEB K, LOTHAR T, MARCO L, et al. Scalable multiobjective optimization test problems. Proc. of the IEEE Congress on Evolutionary Computation, 2002: 825 - 830 .

[17] GÓMEZ R H, COELLO C A C. Improved metaheuristic based on the R2 indicator for many-objective optimization. Proc. of the IEEE Congress on Evolutionary Computation, 2015: 679686.

[18] DEB K. Multi-objective optimization using evolutionary algorithms. New York: Wiley, 2001.

[19] MIETTINEN K, MAKELA M M. On scalarizing functions in multiobjective optimization. OR Spectrum, 2002, 24(2): $193-$ 213.

[20] GOLDBERG D E. Probability matching, the magnitude of reinforcement and classifier system bidding. Machine Learning, 1990, 5: $407-425$.

[21] ZITZLER E, THEIELE L, LAUMANNS M, et al. Performance assessment of multiobjective optimizaers: an analysis and review. IEEE Trans. on Evolutionary Computation, 2003, 7(2): $117-132$.

[22] DEB K, AGRAWAL R B. Simulated binary crossover for continuous search space. Complex Systems, 1995, 9: 115-148.

[23] HUBAND S, HINGSTON P, BARONE L, et al. A review of multiobjective test problems and a scalable test problem toolkit. IEEE Trans. on Evolutionary Computation, 2006, 10(5): $477-506$.

\section{Biographies}

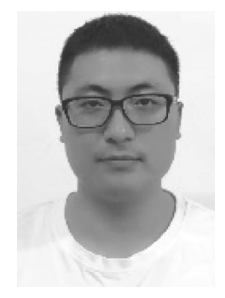

LYU Chengzhong was born in 1990. He received his M.S. degree from Air Force Engineering University (AFEU). He is currently pursuing his Ph.D. degree at AFEU. His research interests include operatonal deployment, operational assessment and decision-making optimization. E-mail: 1101596177@qq.com

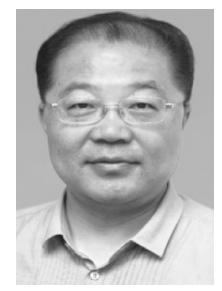

LI Weimin was born in 1964. He received his doctorate from the University of Electronic Science and Technology. His research interests include aerospace defense system construction, command and control planning, natural computation and intelligent information processing. E-mail: liweimin kgd@163.com 